\title{
Fabulation et métalepse dans le diptyque Fangirl (2013) et Carry On (2015) de Rainbow Rowell
}

\author{
Fantasy and Metalepsis in Rainbow Rowell's Diptych Fangirl (2013) \\ and Carry On (2015)
}

RÉSUMÉ

Dans Fangirl (2013) et Carry On (2015), l'écrivaine américaine Rainbow Rowell brouille les frontières entre la réalité et la fiction, ainsi qu'entre le récits extradiégétiques et intradiégétiques. Cet article vise à apporter un éclairage sur les aspects transgressifs et instables des mondes fictifs de Rowell où les personnages sont capables de passer d'un niveau diégétique à un autre. En plaçant 1'histoire enchâssée dans Fangirl à un autre niveau, l'autrice de ce livre pour la jeunesse créé une œuvre où les frontières n'existent pas. A travers l'expansion des mondes entrelacés, elle bouscule les codes standards et normatifs du temps et de l'espace dans la littérature.

Mots-clés : fabulation, métalepse, Rainbow Rowell, littérature américaine, fanfiction

\section{ABSTRACT}

In Fangirl (2013) and Carry On (2015), the American writer Rainbow Rowell blurs the lines between reality and fiction, as well as extradiegetic and intradiegetic narratives. This article aims to shed light on the transgressive and unstable aspects of Rowell's fictional worlds where her characters are able to move from one diegetic level to another. By placing the embedded story in Fangirl at another level, the Young Adult author presents a work where borders do not exist. Through the expansion of the intertwined worlds, she jostles the standard and normative codes of time and space in literature.

Keywords: fabulation, metalepsis, Rainbow Rowell, American literature, fanfiction

\section{Introduction}

- Le but de la fanfiction, expliqua [Cath], c'est de pouvoir jouer avec l'univers de quelqu'un d'autre. D'en réécrire les règles. Ou de les plier selon tes désirs. L'histoire n'a pas à se terminer quand Gemma Leslie s'en lasse. Tu peux rester dans ce monde que tu aimes aussi longtemps que tu le souhaites ; aussi longtemps que tu as de nouvelles histoires à raconter...

- La fanfiction...

1 Texte original : "The whole point of fanfiction," [Cath] said, "is that you get to play inside somebody else's universe. Rewrite the rules. Or bend them. The story doesn't have to end when Gemma Leslie gets tired of it. You can stay in this world, this world you love, as long as you

Marion Velain, Department of French and Italian, Indiana University Bloomington, 355 North Jordan Avenue, Global and International Studies Building, 3rd Floor West Bloomington, IN 47405-1105, mvelain@iu.edu, https://orcid.org/0000-0003-1222-3976 
Briser les règles ou les détourner, c'est ce que fait Cath, une jeune étudiante américaine qui fait ses premiers pas à l'université en essayant de s'éloigner de Wren, sa sœur jumelle, dans Fangirl (2013) de Rainbow Rowell. Fan de la série littéraire Simon Snow de Gemma T. Leslie (GTL) ${ }^{2}$ qui retrace les aventures de ce jeune sorcier, Cath s'est créée une renommée à travers le monde virtuel où elle écrit et poste des chapitres de sa propre fanfiction Carry On, $\mathrm{Simon}^{3}$, fiction homoérotique entre le héros et son némésis, Baz. Le roman de Rowell met en exergue l'état compétitif du personnage, qui souhaite finir sa fanfiction avant que le huitième et ultime opus de la saga littéraire ne sorte en librairie.

Avec un succès timide mais toutefois grandissant du côté francophone, l'autrice fait pourtant preuve d'une très forte popularité chez les adolescents américains. Très rarement étudiée dans le monde universitaire, Rowell n'hésite pas à mettre en avant l'écriture non canonique ainsi que le milieu virtuel, quitte à rester à l'écart de certains auteurs de romans jeunes adultes qui font davantage l'objet de recherche académique.

Fangirl est donc le socle qui va permettre à Rowell d'étendre cet univers fictionnel, et en particulier celui de Simon Snow, qui n'est présenté que partiellement à travers le personnage de Cath. Carry On, roman publié deux ans après Fangirl, en 2015, relate alors l'histoire de Simon Snow et se présente à première lecture comme la fiction terminée de Cath puisque Simon et Baz entrent dans une relation homoérotique, contrairement à la fiction authentique de GTL. Avec ce roman qui se révèle comme pendant de Fangirl, Rainbow Rowell met en place un diptyque très ingénieux qui interroge les différents niveaux de lecture possibles et l'instabilité des frontières ontologiques qui ne cessent d'évoluer et de troubler les limites entre réel et fiction.

L'article examinera la manière dont Rowell met, à certains moments, l'accent sur la fictionnalité de Cath et à d'autres, sa présence au contraire bien réelle comme personne et non comme personnage. Il sera également question d'étudier comment les différents niveaux diégétiques dans Fangirl s'entrelacent et alimentent l'idée d'une littérature transgressive, notamment avec l'intérêt porté à la fabulation et le désir de mettre en tension artificialité et authenticité. Le roman Carry On

want, as long as you keep thinking of new stories "."Fanfiction," Levi said" (Rowell, 2013, p. 127). Les citations extraites des œuvres de Rainbow Rowell, ou certaines sources secondaires, sont des traductions personnelles, nécessaires pour une lecture littérale du texte. Les références données sont celles de l'œuvre originale.

2 Gemma T. Leslie (GTL) est une autrice complètement fictive qui n'apparaît que dans l'univers de Fangirl, tout comme Simon Snow qui n'est qu'une série littéraire fictionnelle que l'on ne peut découvrir qu'à travers le roman de Rowell.

3 Le titre de Carry On, Simon sera utilisé au cours de l'article pour faire référence à la fanfiction de Cath qui est mentionnée dans Fangirl, tandis que Carry On fera directement référence à l'œuvre de Rowell publiée en 2015. 
permettra finalement de prendre précisément du recul et de porter un regard plus large sur l'œuvre de Rowell où la métalepse de la fanfiction originelle dans Fangirl provoque un effet de vertige et offre une représentation instable et audacieuse de la littérature jeune adulte.

\section{Entre fictionnalité et réalité}

Dès le début du second chapitre de Fangirl, Cath est présentée comme un personnage plus vrai que nature qui est en opposition avec les personnages fictifs habituels :

Dans les livres, quand les gens se réveillent dans un endroit qui leur est étranger, ils ont toujours ce moment où ils se sentent désorientés quand ils ne savent pas où ils sont.

Ça n'était jamais arrivé à Cath ; elle se souvenait toujours du moment où elle s'endormait ${ }^{4}$.

Un contraste surprenant s'établit entre les personnages fictifs dans les livres et le personnage de Cath puisqu'elle n'agit pas de la même manière que les personnages de fiction quand ils se « réveillent dans un lieu qui leur est étranger». Cath est introduite au lecteur comme une vraie personne, comme si elle n'était pas faite de papier ; l'expression « dans les livres » indique de façon explicite que Cath est différente, comme si elle ne faisait pas partie d'un roman. Cet étrange effet pourrait donc faire écho aux notions de fabulation et de métafiction.

Dans Fangirl, Rowell joue souvent avec l'ambivalence entre la fiction et la réalité et présente Cath comme un réel être humain qui s'interroge sur la fiction et son fonctionnement. Un paradoxe s'ensuit donc : c'est parce qu'il y a fictionnalité intradiégétique reconnue de son travail que Cath, par contraste, est construite comme une "véritable " personne dans le niveau extradiégétique. Ces récits imbriqués les uns dans les autres fonctionnent à travers des niveaux narratifs différents, comme le suggère Genette, dans Figures III : « tout événement raconté par un récit est à un niveau diégétique immédiatement supérieur à celui où se situe l'acte narratif producteur de ce récit » (Genette, 1972, p. 238). Cath maintient une relation très intense avec la réalité comme si elle en faisait autant partie que le lecteur :

Cath aurait voulu revenir en arrière et réécrire toutes les scènes qu'elle avait écrites sur les torses de Baz et Simon. Elle les avait décrits plats et fermes. Levi était tout en mouvement et en souffle, en courbes et en creux sensuels. Le torse de Levi était une chose vivante ${ }^{5}$.

4 Texte original : "In books, when people wake up in a strange place, they always have that disoriented moment when they don't know where they are.

That had never happened to Cath; she always remembered falling asleep" (Rowell, 2013, p. 14).

5 Texte original : "Cath wanted to go back and rewrite every scene she'd ever written about Baz or Simon's chests. She'd written them flat and sharp and hard. Levi was all soft motion and breath, curves and warm hollows. Levi's chest was a living thing" (Rowell, 2013, p. 421). 
En réfléchissant à la manière dont elle a décrit les corps de ses personnages préférés dans ses fanfictions, Cath ne peut pas s'empêcher de faire la comparaison avec ses propres expériences réelles. La réalité et la fiction sont toujours étroitement liées dans l'œuvre de Rowell, tout comme le buste de Levi qui est présenté comme une chose vivante, bien que le jeune homme reste tout de même un personnage dans un livre qui n'est pas moins fictif que Baz et Simon. Rowell présente ironiquement la fictionnalité comme fabulation ou imagination intradiégétique dès le tout début de Fangirl puisque Cath ne peut pas s'empêcher de penser qu'elle fait semblant d'être dans un film : "Alors qu'elle se rendait à son cours, Cath ne pouvait pas s'empêcher de penser qu'elle jouait le rôle d'une jeune étudiante dans l'un de ces films stéréotypés sur le passage à l'âge adulte $»^{6}$. Cette phrase souligne l'artificialité et la fictionnalisation; en contrastant Cath avec de la fiction, Rowell, paradoxalement, attire en fait l'attention sur la fictionnalité de Cath, car la protagoniste est bien une étudiante universitaire dans un roman d'apprentissage sur le rite de passage à l'âge adulte, elle n'a pas à faire semblant d'être fictive, elle l'est. Cath est en fait la marionnette de Rowell et est alors complètement sous le contrôle de l'autrice. Elle est consciente de sa propre fictionnalité qui ne semble que la naturaliser.

\section{Entrelacs des récits enchâssés}

Ainsi, la fiction de Fangirl est constamment entremêlée avec la fanfiction Carry On, Simon que Cath écrit, mais aussi liée avec la série littéraire authentique créée par GTL. La toute première phrase de Fangirl « Il y avait un garçon dans sa chambre $\gg^{7}$ est répétée dans l'extrait de Simon Snow and the Mage's Heir qui suit le premier chapitre de Fangirl. Par conséquent, Cath semble vivre et faire l'expérience des mêmes circonstances que son personnage préféré. Plus tard dans le roman, les sessions de lecture à haute voix avec le personnage Levi, renforcent l'ambivalence entre la réalité et la fiction. En effet, quand Cath lit une fanfiction à son petit ami, la fiction rattrape la réalité de la vie de Cath : "Cath sentit Levi rire contre sur sa nuque $\rangle^{8}$. Cette phrase est directement suivie par la reprise de la lecture de Cath en italique : "Simon sentit le rire de Baz sur ses cils $»^{9}$. Ces parallèles multiples entre la vie de Cath et sa fanfiction soulignent le besoin incessant d'imaginer et de fictionnaliser constamment les choses jusqu'à glorifier la réalité et la rendre aussi belle que la fiction. Patricia Waugh note que dans le cadre fictionnel, les personnages sont réels à part entière :

6 Texte original : "Walking to class, Cath couldn't shake the feeling that she was pretending to be a college student in a coming-of-age movie." (Rowell, 2013, p. 15).

7 Texte original : "There was a boy in her room." (Rowell, 2013, p. 4).

8 Texte original : "Levi laughed, and Cath felt it on her neck" (Rowell, 2013, p. 399).

9 Texte original: "Baz laughed, and Simon felt it on his eyelashes" (Rowell, 2013, p. 399). 
La fiction est simplement un ensemble différent de " cadres », un ensemble différent de conventions et de constructions. Ainsi, un personnage fictif n'est « pas réel », dans un sens, mais les personnages qui ne sont pas des personnes sont toujours « réels », existent encore à l'intérieur de leurs mondes spécifiques [guillemets de l'auteur cité] ${ }^{10}$.

Cath est alors réelle mais seulement dans le cadre de Fangirl, dans le cadre de son propre monde.

Ce processus d'écriture particulier pourrait précisément faire écho à la vision postmoderne de la fabulation. Le terme, inventé par Robert Scholes dans son livre The Fabulators (1967), a été développé dans Fabulation and Metafiction (1979) : « Les fabulations continuent d'exister, car elles fonctionnent pour les êtres humains comme signes d'une réalité inatteignable, et comme symboles de la difficulté humaine à imaginer cette réalité. Ce sont $[\ldots]$ de vrais rêves $\rangle^{11}$. Dans une certaine mesure, Rowell veut créer de vrais rêves à partir de ses romans ; Cath se perd dans le monde fictionnel de Simon Snow:

Cela faisait un bien fou d'écrire de nouveau dans sa chambre, dans son lit. De se perdre dans le Monde des Mages sans chercher à en partir. De n'entendre dans sa tête que les voix de Simon et de Baz. Même la sienne s'absentait. C'était pour cette raison que Cath écrivait de la fiction. Pour toutes ces heures où leur monde remplaçait la réalité. Quand elle sentait leurs sentiments pour l'un et l'autre déferler sur elle sans s'arrêter ${ }^{12}$.

Selon Scholes (1979), la fabulation nous permet d'accéder à une expérience imaginative, mais il note également la différence entre la fabulation et fabuler : « [la fabulation] doit nous procurer une expérience imaginative nécessaire à notre bien-être créatif [...] Mais alors que notre imagination s'étire et que nous devenons plus sérieux [...] nous n'avons pas besoin de fabuler mais de la fabulation $»^{13}$. Fabuler implique simplement la fiction en elle-même et ce qu'elle révèle directement, tandis que la fabulation est le terme utilisé pour évoquer la façon dont l'histoire est écrite. La fiction est par conséquent une part importante

10 Texte original : "Fiction is merely a different set of "frames", a different set of conventions and constructions. In this view, a fictional character is "unreal" in one sense, but characters who are not persons are still "real", still exist, within their particular worlds" (Waugh, 1984, p. 100).

11 Texte original : "fabulations endure because they continue to function for human beings as signs of some unattainable reality, and as emblems of the human struggle to imagine that reality. They are $[\ldots]$ real dreams" (Scholes, 1979, p. 20).

12 Texte original : "It felt good to be writing in her own room, in her own bed. To get lost in the World of Mages and stay lost. To not hear any voices in her head but Simon's and Baz's. Not even her own. This was why Cath wrote fic. For these hours when their world supplanted the real world. When she could just ride their feelings for each other like a wave, like something falling downhill" (Rowell, 2013, p. 98).

13 Texte original : "[Fabulation] must provide us with an imaginative experience which is necessary to our imaginative well-being [...] But as our imagination stretches and we grow more serious [...] we require not fabling but fabulation" (Scholes, 1979, p. 24). 
de la réalité et a constamment besoin d'être exprimée à travers l'imagination et la fabulation. Avec le personnage de Cath, Rainbow Rowell met l'accent sur la dimension nécessaire de la fabulation et de la fiction.

Dans Fangirl, la coexistence de la fiction et de la réalité est explicitement visible : la présence oppressive de l'univers fictionnel de Simon Snow - plus précisément l'écriture de la fanfiction Carry On, Simon - dans la vie de Cath, est une manière de mêler réalité et fiction ainsi que leur contiguïté puisque la fiction de Simon Snow a un impact sur la vie de Cath. En écrivant autant de fiction, Cath commence à penser et à agir comme les personnages de son histoire. Rowell note qu'il est davantage difficile de vivre la réalité que de la décrire dans la fiction; Cath écrit un bon nombre de scènes entre Baz et Simon mais se rend très vite compte que la réalité est beaucoup plus complexe, qu'elle change et bouge constamment. Le personnage de Cath compare constamment sa relation avec Levi avec la relation de Baz et Simon. Ainsi, la fanfiction de Cath s'entremêle à la vie de la jeune femme et alors qu'elle souligne le fait que la fiction soit meilleure et que la réalité ne fasse pas le poids face à l'imagination, elle utilise, vers la fin du roman, ses comparaisons avec le monde fictionnel de Simon Snow pour illustrer le pouvoir de sa relation avec Levi et la mettre sur le même pied d'égalité que celle de Baz et Simon :

C'était lui le Ballon Rouge. Elle se mit sur la pointe des pieds jusqu'à ce qu'elle fût si proche de son visage qu'elle ne put plus regarder qu'un seul de ses yeux à la fois.

-Tu es magique, lui dit-elle [italiques de l'auteur cité] ${ }^{14}$.

Le but de ces mots accentués par les italiques est de marquer la distinction entre la fiction et la réalité : Simon Snow, dans les livres authentiques de GTL, avait toujours une petite balle rouge en sa possession quand il était petit, ce qui représentait la magie (le lecteur l'apprend dans Carry On de Rainbow Rowell, où la petite balle est mentionnée et les personnages donnent plus de détails à son $\operatorname{propos}^{15}$ ). En associant Levi à la magie, Cath partage explicitement son attirance envers l'imaginaire et la fabulation. Morace, dans un essai sur Fabulation and Metafiction, cite Scholes $(1967,1979)$ pour parler de fabulation comme « fantasme éthiquement contrôlé $»^{16}$. Ce fantasme contrôlé signifie donc principalement ressentir de la joie en conceptualisant des structures et en développant des idées.

14 Texte original : "He was The Red Balloon. She lifted her heels and pulled him forward until his face was so close, she could look at only one of his eyes at a time. 'You're magic,' she said" (Rowell, 2013, p. 433).

15 Dans Carry On de Rowell, Simon se bat contre le Indidious Humdrum, le méchant espiègle qui a le même physique que Simon lorsqu'il avait onze ans et qu'il est arrivé à Watford, l'école des sorciers : «[Il] ressemble à [Simon]. Parle comme [lui]. Jette cette balle rouge infernale dans les airs comme [lui] » (Rowell, 2015, p. 420).

16 Texte original : "Fabulation includes a certain didactic quality and constitutes what Scholes calls an "ethically controlled fantasy"” (Morace, 1980, p. 370). 
La fiction n'a pas entièrement le projet de représenter la réalité, elle implique également l'expression du pouvoir des mots pour stimuler l'imagination.

Comme le mentionne le titre du livre de Scholes (1979), la métafiction ${ }^{17}$ fait partie de la fabulation. L'auteur est conscient de la fictionnalité de son ou ses personnages et le souligne de façon explicite. La fin de Carry On (2015) reconnait sa dimension métafictionnelle :

[Simon] me regarde dans les yeux.

- Tu es encore Simon Snow. Tu seras toujours le héros de cette histoire...

- Ce n'est pas une histoire !

- Tout est une histoire. Et tu es le héros. Tu as tout sacrifié pour moi [italiques de l'auteur cité] ${ }^{18}$.

En expliquant que Simon est toujours le héros de l'histoire par l'intermédiaire de Baz, Rowell met l'accent sur le caractère fictionnel de ses propres œuvres. En fait, Simon et Baz sont présentés comme personnages fictifs dans Carry On beaucoup plus facilement que le sont Levi et Cath dans Fangirl par exemple, comme s'il était nécessaire et évident de souligner et rappeler la fictionnalité de Carry On à cause de son niveau intradiégétique dans Fangirl. Baz et Simon sont présentés comme personnages fictifs dans Fangirl, ce qui n'est pas le cas concernant Levi et Cath. Ainsi, Carry On met explicitement l'accent sur la fictionnalité de ces personnages, parce qu'ils font tout simplement partie, à l'origine, d'une histoire. La métafiction explore la relation entre la fiction et la réalité : le statut ontologique d'un personnage est donc interrogé. Les personnages fictifs à la fois existent et n'existent pas ; par exemple, comme on a pu le constater précédemment, Cath est présentée comme personne réelle par rapport à Simon et Baz dans Fangirl, bien qu'elle soit tout autant fictive qu'eux. Patricia Waugh écrit :

[La plupart des auteurs métafictionnels] sont nerveux et gênés d'affirmer que, bien que la fiction littéraire soit seulement une réalité verbale, elle construit à travers le langage un monde imaginatif qui a, en ces propres termes, un statut référentiel total comme une alternative au monde dans lequel nous vivons ${ }^{19}$.

17 Selon le dictionnaire Oxford English Dictionary, ajoutez le dictionnaire à la bibliographie avec la page de la citation la définition de la métafiction (théorie littéraire) est : "Fiction in which the author self-consciously alludes to the artificiality or literariness of a work » [Une fiction dans laquelle l'auteur fait allusion de façon auto-référentielle à l'artificialité ou la litérarité d'une œuvre].

18 Texte original : "[Simon] meets my eyes. "You're still Simon Snow. You're still the hero of this story -

This isn't a story!

Everything is a story. And you are the hero. You sacrificed everything for me (Rowell, 2013, p. 507).

19 Texte original: "[Most metafictional writers] are self-consciously anxious to assert that, although literary fiction is only a verbal reality, its constructs through language an imaginative world that had, within its own terms, full referential status as an alternative to the world in which we live" (Waugh, 1984, p. 100). 
Carry On se réfère à sa propre fictionnalité à cause de sa dimension déjà fictionnelle dans le roman précédent tandis que Fangirl attire l'attention sur un «monde alternatif».

\section{Métalepse transgressive}

Le diptyque de Rowell, Fangirl et Carry On, se repose donc sur la structure de " l'histoire dans l'histoire » ou plus communément appelés les récits enchâssés. Originellement implanté dans Fangirl, Carry On est devenu de manière autonome un spin-off littéraire.

Carry On, Simon, la fanfiction de Cath, est l'histoire implantée dans Fangirl: cependant, Carry On, le roman de Rowell, ne fait plus partie de Fangirl mais obtient sa propre dimension ontologique et référentielle et devient par conséquent une œuvre métaleptique. Gérard Genette définit la métalepse ainsi : «toute intrusion du narrateur ou du narrataire extradiégétique dans l'univers diégétique (ou de personnages diégétiques dans un univers métadiégétique, etc.), ou inversement » (Genette, 1972,p. 244). Quand les frontières deviennent poreuses et que les niveaux narratifs se croisent, on peut remarquer un effet métaleptique ; la transgression est la caractéristique la plus importante de la métalepse. Carry On, en ayant sa propre dimension diégétique, sort de son premier niveau intradiégétique :

La métalepse ontologique est plus qu'un clin d'œil furtif qui perce les niveaux, c'est un passage logiquement interdit, une transgression qui permet l'interpénétration de deux domaines censés rester distincts. Cette opération remet radicalement en question la frontière entre imaginaire et réel. On pourrait comparer [...] la métalepse ontologique à une croissance envahissante qui détruit la structure de ces tissus (Ryan, 2005, p. 207).

Définir le roman de Rowell comme métaleptique n'est seulement possible que lorsque le cadre structurel du diptyque est pris en compte : la fanfiction dans le monde fictionnel de Fangirl devient une véritable histoire que le lecteur dans le monde réel peut lire, elle ne se passe plus seulement dans le monde fictionnel mais a sa propre matérialité. Carry On est passé du monde fictionnel et imaginaire au monde réel et tangible.

Cependant, en transgressant cette limite, Carry On perd sa connexion avec son autrice initiale, Cath; Carry On, le roman de Rowell, ne dépend plus du personnage fictif, car il est sorti de ce monde fictionnel original. Dans la note de l'autrice à la fin de Carry On, Rowell explique que c'est elle l'autrice de ce roman :

Si vous avez lu mon livre Fangirl, vous savez que Simon Snow a commencé en tant que personnage fictif dans ce roman.

Un personnage fictif-fictif. Presque comme un amalgame et un héritier d'une centaine d'autres Elus fictifs.

$[\ldots]$

J'ai lu et adoré tellement d'histoires magiques sur les Elus : comment écriais-je la mienne? 
C'est ce qu'est Carry On.

C'est ma vision d'un personnage que je ne pouvais pas me sortir de la tête. C'est ma vision de ce genre de personnage, et ce genre de parcours initiatique.

C'était ma façon à moi de donner à Simon et Baz, seulement à moitié imaginés dans Fangirl, l'histoire que je pensais leur devoir [italiques de l'auteur cité] ${ }^{20}$.

Baz et Simon, les personnages intradiégétiques du récit enchâssé dans Fangirl, définis comme « fictifs-fictifs » par Rowell, ont donc traversé un niveau diégétique puisque dans Carry On, ils montent d'un niveau, ils ne sont plus que fictifs ; Rowell les retire du monde fictionnel de Fangirl pour leur donner leur propre fictionnalité dans un roman qui peut être vu comme le leur, dans leur propre univers ontologique. Ainsi, les personnages de Carry On passent d'un niveau diégétique à l'autre ; ils vont d'un monde à l'autre.

Rowell a tendance à manipuler les personnages et les mondes fictionnels : Landline $^{21}$, un de ses romans, comporte un caméo ${ }^{22}$ de Levi et Cath à la fin du livre. Selon Rowell, Carry On est son histoire tandis que la fanfiction de Cath ne peut pas être considérée comme la sienne, car elle ne lui appartient pas complètement puisque Cath se positionne comme intermédiaire entre l'autrice et l'histoire. Carry On est l'idée que Rowell se fait des personnages, l'inspiration qu'elle en tire, ce qui lui permet alors de narrer l'histoire qu'elle souhaite créer pour eux. Bien que Cath soit son personnage et donc sa création, tout autant que l'est la fanfiction de la protagoniste, Rowell définit une limite précise entre le monde fictionnel de Fangirl et celui de Carry On; le Carry On, Simon de Cath n'est pas le Carry On de Rainbow Rowell. Le roman de 2015 ne recycle pas les fragments divers de Carry On, Simon qui se trouvent entre chaque chapitre de Fangirl. Chaque petit extrait dans Fangirl n'existe seulement que dans le monde de Cath ; ainsi, il est

20 Texte original : "If you've read my book Fangirl, you know that Simon Snow began as a fictional character in that novel.

A fictional-fictional character. Kind of an amalgam and descendant of a hundred other fictional Chosen Ones.

I've read and loved so many magical Chosen One stories - how would I write my own?

That's what Carry On is.

It's my take on a character I couldn't get out of my head. It's my take on this kind of character, and this kind of journey.

It was a way for me to give Simon and Baz, only half-imagined in Fangirl, the story I felt I owed them" (Rowell, 2015, Author's note).

${ }^{21}$ Landline (2014) se déroule dans le Nebraska, tout comme dans Fangirl.

22 Caméo, de l'italien "cammeo », est souvent utilisé dans le milieu cinématographique et traduit l'idée d'une apparition brève d'un personnage fictif dans une autre œuvre d'art. Cath et Levi font partie du récit de Landline bien que leurs prénoms ne soient pas mentionnés. Rowell utilise plusieurs notions cinématographiques, comme le caméo ou le sppin-off dans son œuvre littéraire et écrit presque une fresque avec ses romans où chaque monde se rejoint et est connecté à l'autre. Cath and Levi voyagent d'un roman à l'autre parce qu'ils sont sur le même niveau ontologique que les personnages de Landline et se trouvent au même endroit (Nebraska). 
seulement à moitié imaginé, car le lecteur n'a accès qu'à des aperçus du monde de Simon Snow. La fanfiction dans Fangirl ne peut jamais être complète car sa dimension ontologique est intradiégétique.

En outre, la fanfiction en général peut être considérée métaleptique, car Cath incarne la fangirl qui s'immerge dans la fiction et devient alors créatrice et non plus seulement lectrice, comme l'explique Turk (2011) :

Comme les vidéos, les fanfictions sont généralement métaleptiques dans le sens où leur existence réside dans la trace textuelle des spectateurs s'immergeant dans un monde fictionnel, ces derniers devenant créateurs pour transformer des histoires déjà écrites. [...] À travers la fanfiction, donc, les fans passent de membres du public à auteurs et directeurs afin de (ré) écrire ou (re)diriger une histoire selon leur propre vision. Encore une fois, le public prend le contrôle du discours : les auteurs de fanfiction présentent leurs propres interprétations et désirs (ainsi que, dans certains cas, leurs désirs sexuels) dans un récit partagé ; puisqu'il y a un changement de médium, les lecteurs de fanfiction doivent être des participants actifs en ornant ces histoires de sens (p. 95).

Non seulement le roman Carry On est métaleptique dans son cadre structurel puisqu'il devient un livre réel et matériel, mais Carry On, Simon, la fanfiction dans Fangirl est également métaleptique puisque Cath utilise un texte authentique pour en faire le sien et devenir autrice. Cependant, dans le cas de Cath, cela est plus complexe parce que la fanfiction qu'elle écrit n'a pas d'origine canonique préexistante à l'extérieur du monde fictionnel. Fangirl est seulement la représentation d'une structure métaleptique concernant la fanfiction, car la série de Gemma T. Leslie n'existe pas.

\section{Conclusion}

Avec une telle structure enchâssée qui finit par se déployer et s'ouvrir complètement, le diptyque Fangirl et Carry On met en lumière l'instabilité des frontières entre réel, imaginaire et fiction, et souligne l'idée que ce mouvement entre les niveaux peut parfois se révéler transgressif.

Le personnage de Cath dans Fangirl, pur intermédiaire entre les divers niveaux diégétiques, le réel et la fiction, mais aussi l'autrice et son œuvre, montre la complexité de l'échange entre auteur et fiction lorsqu'il y a réécriture d'une œuvre écrite par le même auteur. Cath, malgré le fait que Rowell estampille Carry On comme son œuvre et non celle de son personnage, est toujours présente dans l'imaginaire du lecteur de Carry On : en effet, Carry On porte non seulement le nom de la fanfiction de Cath mais reprend l'histoire homoérotique entre Baz et Simon. Ce diptyque des deux romans de Rowell montre à quel point les frontières sont instables et met en lumière comment l'espace du texte est occupé de différentes manières, et n'est jamais complètement saisissable. 


\section{References}

Genette, G. (1972). Figures III. Paris: Seuil.

Morace, R A. (1980). On "Fabulation and Metafiction". Studies in the Novel, 12(4), 369-374.

Oxford English Dictionary. Retrieved November 1, 2020, from https://www.oed.com.

Rowell, R. (2013). Fangirl. New York: St. Martin's Press.

Rowell, R. (2014). Landline. New York: St Martin's Press.

Rowell, R. (2015). Carry On. New York: St Martin's Press.

Ryan, M.-L. (2005). Logique culturelle de la métalepse, ou la métalepse dans tous ses états. In J. Pier, \& J.-M. Schaeffer (Eds.), Métalepses. Entorses au pacte de la représentation (pp. 201223). Paris: École des hautes études en sciences sociales.

Scholes, R. (1967). The Fabulators. New York: Oxford University Press.

Scholes, R. (1979). Fabulation and Metafiction. Urbana: University of Illinois Press.

Turk, T. (2011). Metalepsis in Fan Vids and Fan Fiction. Metalepsis in Popular Culture, 28, 83-103. DOI: $10.1515 / 9783110252804.83$.

Waugh, P. (1984). Metafiction. The Theory and Practice of Self-Consicous Fiction. London, New York: Routledge. 